\title{
Heart-on-a-Chip Model for Cardiomyopathies
}

Editorial

Joshi-Mukherjee $\mathrm{R}^{*}$

Research Scientist, Aurora Health Care, Center for Integrative Research on Cardiovascular Aging, (CIRCA), Aurora Research Institute ${ }^{\mathrm{TM}}$, Milwaukee, WI, USA.

Cardiovascular disease is the number one cause of death in the US. According to US Center for Disease Control and Prevention (CDC), about 800,000 American deaths is attributed to cardiovascular disease and each year about hundreds of thousands of people die well before the current life expectancy of 78 years in the US. By 2030, approximately $20 \%$ of the population will be aged 65 or older and cardiovascular diseases will be the leading cause of death. Over the past 4 decades, there have been remarkable advances in our understanding and treatment of ischemic heart disease, while our understanding of cardiomyopathies and arrhythmic disorders has lagged behind. Indeed, the realization that many cardiomyopathies and sudden cardiac deaths are in fact related to genetic disorders has heralded a remarkable era for hereditary cardiac disorders. With advances in genetic sequencing methods, more genetic targets are being identified as pathogenic variants of these disorders. Unfortunately, understanding the biology of how these putative genetic variants result in observed phenotypic changes has proven challenging. The patient harbors the mutations from birth but the onset of the development of rhythm disturbances presents much later in life thus indicates the requirement of higher order cardiac model that will encompass patient bio-omics. Genetic variation contributing to complex traits poses numerous barriers in facilitating the development of precision medicine. Classically, various non-human in-vivo models of gain- or loss-of-function of a gene have been central to the elucidation of physiological or developmental function. However, increasing number of drugs fail to replicate in human clinical trials posing looming challenges for human disease modeling. A big hurdle to understanding cardiac molecular and cellular physiology is the inability to maintain and study adult human cardiomyocytes in culture. Furthermore, the invasive nature of obtaining primary human cardiac tissue has hindered the field compared with other fields such as hematology or oncology. This leaves scientists to rely on animal models or transfected cell models. Historically, cardiovascular safety testing relied on in vivo models to replicate the integrated nature of cardiovascular physiology. While in vivo models offer good translation for positive findings, they have several limitations namely, 1) the action potential of rodents is significantly shorter and lacks phase-2 plateau; 2) they lack mature $\beta$-adrenergic signaling and excitation-contraction (E-C) coupling; and 3 ) these models for the safety evaluation of candidate drugs are cost-intensive, time-consuming and are often insufficient to predict human relevant cardiotoxicity and arrhythmia. Human induced pluripotent stem cells (hiPSCs) have emerged as a reliable method of producing patient-specific somatic tissue lines via directed differentiation. hiPSC derived beating heart cells from patients' tissue closely mimic adult heart cells that can be used as a model to study abnormal heart mechanical and electrical function. The model has already proven complementary or superior when compared with transfected cell or animal models of cardiac genetic disorders.

\section{Induced Pluripotent Stem Cell Technology and Cardiomyopathies}

Seminal work done by John Gurdon in 1962 to generate entire fertile Xenopuslaeyis by intestinal cells' nuclear transfer to enucleated eggs; generation of Dolly sheep the first mammal to be cloned (Campbell et al., 1996) [1,3]; to more recently reprogramming of somatic cells to an ES cell-like state known as iPSC (Takahashi and Yamanaka, 2006) [4, 5] opened new avenues for targeted therapies that can bring incredible revolution in personalized medicine [6]. The use of hiPSCs to address pathophysiological questions has exploded in recent decade since the discovery of Yamanaka factors [5]. A patient-specific cell with a clinical phenotype allows the investigator to capture and immortalize the individual genome in a dish to probe for crucial genotype-phenotype interactions contributing to the patient pathological conditions [7-9]. hiPSC technology allowed generation of indefinite numbers of patient - specific cells as invaluable tools for developmental biology, human disease model for drug discovery and genetic engineering for personalized therapy. hiPSCs derived from somatic tissue such as

*Corresponding Author:

Rosy Joshi-Mukherjee,

Research Scientist, Aurora Health Care, Center, for Integrative Research on Cardiovascular Aging, (CIRCA), Aurora Research Institute ${ }^{\mathrm{TM}}$, Milwaukee, WI, USA

Email: rosy.joshi-mukherjee@aurora.org

Received: February 24, 2017

Published: March 03, 2017

Citation: Joshi-Mukherjee R (2017) Heart-on-a-Chip Model for Cardiomyopathies. Int J Stem Cell Res Transplant. 5(1e), 1-3. doi: http://dx.doi.org/10.19070/2328-3548-1700011e

Copyright: Joshi-Mukherjee $\mathbf{R}^{\circ}$ 2017. This is an open-access article distributed under the terms of the Creative Commons Attribution License, which permits unrestricted use, distribution and reproduction in any medium, provided the original author and source are credited. 
skin, blood, hair or urine, have unlimited self-renewing and pluripotent capabilities. In 2009, Thomson's lab demonstrated that hiPSCs can be efficiently stimulated to differentiate into cardiac lineage to produce beating cardiomyocytes (hiPSC-CM) [10, 11] that have proven to be a robust platform for the study of several genetic cardiac disorders and drug screening for proarrhythmic risk. To date, iPSC-CMs have been used to successfully model arrhythmic disorders, showing excellent recapitulation of cardiac channel function and electrophysiological features of long (LQT) types 1, 2, 3, and 8, and catecholaminergic polymorphic ventricular tachycardia [12-14]. Additionally, iPSC-CMs have shown some features of the respective phenotypes for arrhythmogenic right ventricular dysplasia/cardiomyopathy, LEOPARD syndrome, Pompe's disease, and Friedriech's ataxia. Similarly, iPSC-CM models of dilated cardiomyopathy and hypertrophic cardiomyopathy have shown robust correlation of predicted morphologic, contractile, and electrical phenotypes [13, 14]. Action potential prolongation is a hallmark of hypertrophied or failing ventricular myocardium and the individual variability of the functional expression of the same genetic defects has created an opportunity for modeling the disease in hiPSCs. A number of studies have used hiPSC to model LQTS and recapitulated the LQTS phenotype and have facilitated insights into the mechanisms of loss of function in patients with $\mathrm{Na}, \mathrm{K}$ and $\mathrm{Ca}$ channel mutations. The life threatening manifestation of cardiac arrhythmia associated with aforementioned cardiac disorders is not just cellular phenomena but is the result of abnormalities in the network properties of the heart. hiPSC derived cardiomyocyte model will help to understand the influence of the variation of genotype among individuals harboring the same mutation on cardiac dysfunction which can then help inform an individual's risk for a potentially lethal arrhythmia [7, 15].

\section{Cancer Drug Induced Premature Cardiomyopa- thies}

Cancer is diagnosed in $>12000$ children and adolescents in the United States each year. Progress in cancer therapeutics over the past 40 years has remarkably improved survival rates for most childhood malignancies. In the United States, this success translates into $>325000$ survivors of childhood cancer, of whom $24 \%$ are now $>30$ years from diagnosis. Tyrosine kinase-targeted therapies have revolutionized the treatment of a variety of cancers. Targeted inhibitors of kinases have improved antitumor efficacy and have fewer toxic side-effects, compared to traditional chemotherapy, but have been implicated in causing serious adverse cardiac events [16-18]. Pediatric cancer patients when treated with approved tyrosine kinase inhibitors (TKIs), present unanticipated cardiac dysfunction that includes QT prolongation, reduction in left ventricle ejection fraction, symptomatic congestive heart failure (CHF) and acute coronary syndrome, and myocardial infarction [19-21]. Precious little is known about the mechanism of TKIs mediated cardiotoxicity and arrhythmia. Left ventricular dysfunction does not seem to be common to all TKIs. Thus, each agent must be understood on a case-by-case basis, focusing on the specific kinases inhibited. In addition, many TKI targets are not known to be expressed in the heart (eg, RET, FLT3, CSF-1R), and therefore cardiotoxicity is unlikely with agents targeting these kinases. However, there are a large number of TKIs on the horizon with targets that are expressed in the heart, for which we do not have good prospective data concerning cardiac effects. hiPSC derived cardiac models will aid in delineating TKIs mediated cardiotoxicity leading to rhythm disorders directed at popular kinase targets in cancer. Delineating the relevance of action potential and calcium transients as early signatures of TKIs mediated myocyte or fibroblast remodeling leading to cardiotoxicity and arrhythmia will advance our knowledge about the role of TKI in cardiac physiology. Confirmation of these potential mechanisms would substantially deepen our basic understanding of cardiotoxicity and arrhythmia in children treated for cancer. Broadly speaking, the role of TKIs on cardiomyocyte or fibroblast commitment would offer new insights in cardiomyocyte development in heart.

\section{Modeling proarrhythmic Risk using Heart-on-a- Chip}

Shortening of repolarization (field potential duration) similar to QT interval recorded on electrocardiogram have been utilized by researcher to monitor the electrophysiological dynamics of multicellular heart-on-a-chip preparation [22-24] thus facilitating cell-based high throughput drug screens which is limiting with other primary human cell lines or animal models. Non-invasive multielectrode array (MEA) mapping technique to measure field potential shows that hiPSC-CMs respond appropriately to compounds known to affect cardiac chronotropy, repolarization and QT intervals properties that are important to identify cardiac arrhythmias in clinical practice $[9,24]$. There is large number of novel cancer or non-cardiac drugs that may pose potential cardiac hazard based on genetic variation due to polymorphism or modifier loci, is hard to predict without functional studies. The autonomic nervous system plays a pivotal role in the regulation of heart rate and contractility. hiPSC-CMs cultured on MEA chip have shown neurohormonal response resulting in an increase in beating frequency and field potential duration shortening [25]. In recent years MEA assay has become well accepted platform for testing drug effectiveness and for arrhythmia screening [25]. This high throughput system holds great potential for discovering novel targets and reducing drug development costs by early screening for cardiotoxicity and dysfunction.

In summary heart-on-a-chip model will significantly advance in our understanding of the biology of cardiac diseases and improve heart function in patients with heart disease causing progressive mechanical or electrical dysfunction otherwise requiring surgical intervention such as ventricular assist device or heart transplantation or implantable defibrillators. Recreating disease phenotype from patient samples in a dish will help in not only understanding the pathobiology of the disease but also in drug discovery. "Heart-on-a-chip model" derived from induced pluripotent stem cell derived cardiomyocytes will help to investigate the mechanism of drug induced cardiotoxicity, muscle disorder and arrhythmogenesis in young and aging population.

\section{References}

[1]. Campbell KH, Alberio R, Choi I, Fisher P, Lee JH, et al., (2005) Cloning: eight years after Dolly. Reprod Domest Anim. 40(4): 256-68.

[2]. Campbell KH, Loi P, Wilmut I, Otaequi PJ (1996) Cell cycle co-ordination in embryo cloning by nuclear transfer. Rev Reprod. 1(1): 40-6.

[3]. Campbell KH, McWhir J, Ritchie WA, Wilmut I (1996) Sheep cloned by nuclear transfer from a cultured cell line. Nature. 380(6569): 64-6.

[4]. Takahashi K, S Yamanaka (2006) Induction of pluripotent stem cells from mouse embryonic and adult fibroblast cultures by defined factors. Cell. 126(4): 663-76. 
[5]. Takahashi K, Tanabe K, Narita M, Ichisaka T, Tomoda K, et al., (2007) Induction of pluripotent stem cells from adult human fibroblasts by defined factors. Cell. 131(5): 861-72.

[6]. Sayed N, C Liu, JC Wu (2016) Translation of Human-Induced Pluripotent Stem Cells: From Clinical Trial in a Dish to Precision Medicine. J Am Coll Cardiol. 67(18): 2161-76.

[7]. Shinozawa T, Nakamura K, Shoji M, Kimura M, Ueda H, et al., (2016) Recapitulation of Clinical Individual Susceptibility to Drug-Induced QT Prolongation in Healthy Subjects Using iPSC-Derived Cardiomyocytes. Stem Cell Reports. 8(2): 226-234.

[8]. Zanella F, F Sheikh (2016) Patient-Specific Induced Pluripotent Stem Cell Models: Generation and Characterization of Cardiac Cells. Methods Mol Biol. 1353: 147-62.

[9]. Kuusela J, Kim J, Rasanen E, Aalto-Setalal K (2016) The Effects of Pharmacological Compounds on Beat Rate Variations in Human Long QT-Syndrome Cardiomyocytes. Stem Cell Rev. 12(6): 698-707.

[10]. Zhang J, Wilson GF, Yu J, Thomson JA, Kamp TJ, et al., (2009) Functional cardiomyocytes derived from human induced pluripotent stem cells. Circ Res. 104(4): e30-41.

[11]. Ma J, Guo L, Fiene SJ, Anson BD, Kamp TJ, et al., (2011) High purity human-induced pluripotent stem cell-derived cardiomyocytes: electrophysiological properties of action potentials and ionic currents. Am J Physiol Heart Circ Physiol. 301(5): H2006-17.

[12]. Limpitikul WB, Dick IE, Tester DJ, Yue DT, Kanter Rj, et al., (2017) A Precision Medicine Approach to the Rescue of Function on Malignant Calmodulinopathic Long QT Syndrome. Circ Res. 120(1): 39-48.

[13]. Jiang W, F Lan, H Zhang (2016) Human Induced Pluripotent Stem Cells for Inherited Cardiovascular Diseases Modeling. Curr Stem Cell Res Ther. 11(7): 533-41.

[14]. Burridge PW, Diecke S, Matsa E, Sharma A, Wu H, et al., (2016) Modeling Cardiovascular Diseases with Patient-Specific Human Pluripotent Stem Cell-Derived Cardiomyocytes. Methods Mol Biol. 1353: 119-30.

[15]. Gao L, Kupfer M, Jung J, Yang L, Sie Y, et al., (2017) Myocardial Tissue Engineering With Cells Derived from Human Induced-Pluripotent Stem
Cells and a Native-Like, High-Resolution, 3-Dimensionally Printed Scaffold. Circ Res. pii: CIRCRESAHA.116.310277.

[16]. Thavendiranathan P, MT Nolan (2017) An emerging epidemic: cancer and heart failure. Clin Sci (Lond). 131(2): 113-121.

[17]. Narayan HK, French B, Hyman D, Smith AM, Ky B, et al., (2017) Detailed Echocardiographic Phenotyping in Breast Cancer Patients: Associations with Ejection Fraction Decline, Recovery, and Heart Failure Symptoms over 3 Years of Followup. Circulation. pii: CIRCULATIONAHA.116.023463

[18]. Ghosh AK, JM Walker (2017) Cardio-oncology. Br J Hosp Med (Lond). 78(1): $\mathrm{C} 11-\mathrm{C} 13$

[19]. Vargas-Neri JL, Castelan-Martinez OD, de jesus Estrada-Loza M, RivasRuiz R, Betanzos-Cabrera Y, (2016) [Anthracycline-induced cardiotoxicity: report of fatal cases]. Rev Med Inst Mex Seguro Soc. 54(3): 404-8.

[20]. Ehrhardt MJ, JM Fulbright, SH Armenian (2016) Cardiomyopathy in Childhood Cancer Survivors: Lessons from the Past and Challenges for the Future. Curr Oncol Rep. 18(4): 22.

[21]. Lipshultz SE, Kutok JL, Miller TL, Colan SD, Kelly KM, et al., (2013) Impact of hemochromatosis gene mutations on cardiac status in doxorubicin-treated survivors of childhood high-risk leukemia. Cancer. 119(19): 3555-62.

[22]. Del Alamo JC, Lemons D, Serrano R, Bodmer R, Mercola M, et al., (2016) High throughput physiological screening of iPSC-derived cardiomyocytes for drug development. Biochim Biophys Acta. 1863(7): 1717-27.

[23]. Clements M (2016) Multielectrode Array (MEA) Assay for Profiling Electrophysiological Drug Effects in Human Stem Cell-Derived Cardiomyocytes. Curr Protoc Toxicol. 68: 22 4 1-22 432.

[24]. Sallam K, Li Y, Sager PT, Houser SR, Wu JC (2015) Finding the rhythm of sudden cardiac death: new opportunities using induced pluripotent stem cell-derived cardiomyocytes. Circ Res. 116(12): 1989-2004.

[25]. Navarrete EG, Liang P, Lan F, Wu JC, Gong T, et al., (2013) Screening drug-induced arrhythmia [corrected] using human induced pluripotent stem cell-derived cardiomyocytes and low-impedance microelectrode arrays. Circulation. 128(11 Suppl 1): S3-13. 